\title{
LA INTRODUCCIÓN DE LA GENÉTICA EN MÉXICO: LA GENÉTICA APLICADA AL MEJORAMIENTO VEGETAL
}

\author{
Ana Lilia Gaona Robles y Ana Barahona Echeverría \\ Facultad de Ciencias, Universidad Nacional Autónoma de México.
}

\section{RESUMEN}

El objetivo de este artículo es estudiar la introducción, sensu Conry, de la genética en México. Para ello, hablaremos de la comunidad médica del XIX, la que desarrolló los principios fundamentales de la «herencia», sin aplicar los principios elementales de la genética. Trataremos de mostrar cómo los principios genéticos fueron introducidos vía las técnicas y no vía la teoría. La investigación genética en México comenzó a través de sus aspectos más prácticos, la genética experimental aplicada al mejoramiento de las especies con intereses económicos.

PALABRAS CLAVE: agronomía, genética aplicada, herencia, México, siglo XIX.

\section{SUMMARY}

The aim of this paper is to deal with the introduction, sensu Conry, of genetics in Mexico. In the XIX Century the medical community was one of the most prominent groups that developed the field of «heredity», although they never used the genetical principles in their field. We will try to show that one of the main events in the history of genetics in Mexico deals with the introduction of techniques, rather than theories. The research in genetics began through one of the most practical applications, experimental genetics applied to the improvement of species with economical interests.

KEY WORDS: agronomy, genetics applied, heredity, México, $19^{\text {th }}$ century.

\section{INTRODUCCIÓN}

La historia de la ciencia está lejos de ser un área de estudio nueva en nuestro país ${ }^{1}$. Aunque en el siglo pasado existen ya diversos autores que se ocupan de hacer biogra-

1 Sería difícil mencionar todos los trabajos que se han hecho sobre historia de las ciencias en México. Sin embargo, es útil recordar que desde el siglo pasado las revistas científicas como La Naturaleza o la Gaceta médica, incluían de manera especial, las biografías de sus integrantes como homenaje póstumo, así como diversos artículos sobre el desarrollo de las instituciones. 
fías de científicos anteriores a ellos y, del seguimiento temporal de instituciones o documentos, no es sino hasta hace unas cuantas décadas cuando esta actividad ha tenido un incremento sensible.

En cuanto a las ciencias biológicas, la mayor parte de los estudios han sido dedicados a la medicina y la botánica, un poco menos a la zoología y a la biología en general, y muy poco o nada, a otras disciplinas como la embriología, la ecología, la paleontología o la genética ${ }^{2}$

Sin embargo, el carácter de casi la totalidad de estos trabajos responde más a visiones biográficas o descripciones históricas que ven los desarrollos científicos como el resultado menos evolucionado de lo que ha acontecido en otras partes del mundo, principalmente Europa y Estados Unidos, que a análisis epistemológicos o metodológicos del contexto local como elemento central de la explicación de la actividad científica en cada región.

Hasta muy recientemente, los estudios históricos «periféricos», con énfasis en el contexto «local», no se han desarrollado en Latinoamérica ${ }^{3}$. El presente trabajo responde a esta postura metodológica.

En éste pretendemos mostrar cómo y cuándo fue introducida la genética en México; es decir, nuestro interés está dirigido hacia la ubicación del momento en que por primera vez se utilizan los principios genéticos dentro de los programas de investigación en México, naturalmente, en el siglo XX.

Requerimos, sin embargo, aclarar lo que en este trabajo se entiende como «introducción». Según Conry la «introducción» de una ciencia la podemos localizar en varios niveles: el primero es el nivel intelectual, en donde la ciencia en cuestión se discute pero no se ejerce; el segundo, se refiere al nivel político, donde la ciencia se utiliza ideológicamente pero no se practica; y el tercero, que es el nivel propiamente científico $^{4}$. En este último nivel no es necesario solamente que la ciencia —en este

2 Revisiones de considerable extensión en la ciencia mexicana general durante el siglo pasado y en el presente son: la obra en tres volúmenes de PARRA, P. (1886-1888), Historia de la medicina en México, desde la época de los indios hasta el presente; el libro de GORTARI, E. (1963), La ciencia en la historia de México, y la obra en cuatro volúmenes de TRABULSE, E. (1989), Historia de la Ciencia en México. Eventos importantes han sido: el Primer Coloquio Mexicano de Historia de la Ciencia (1963) y la formación de la Sociedad Latinoamericana de Historia de la Ciencia y la Tecnología (en la misma década).

3 Véase Vessuri, H. (1997), «Introducción» en: AlbornOZ, M. P. KREIMER, y E. GLAVICH (eds.), (1997), Ciencia y Sociedad en América Latina, Universidad Nacional de Quilmes; VARSAVSKY, O. (1969), Ciencia, política y cientificismo, Buenos Aires, CEAL; LEITE LOPES, J. (1972), La Ciencia y el dilema de América Latina: dependencia o liberación, México, Siglo XXI; HerRERA, A. (1971), Ciencia y política en América Latina, México, Siglo XXI; CUETO, M. (1989), «Excelencia científica en la periferia. Actividades científicas e investigación biomédica en Perú, 1890-1950, Lima, GRADE-CONCYTEC; Cueto, M. «Laboratory Styles in Argentine Physiology», Isis, 85, 2, junio; GLICK, T. F. (1992), «Science and Society in Twentieth-Century Latin America», en: BeTHELl, L. (ed.), The Cambridge History of Latin America, vol. VI, Cambridge, Cambridge University Press. 1992.

4 CONRY, I. (1974), L'introduction du Darwinisme en France au XIXe siècle, París, Ed. Vrin. 


\section{LA INTRODUCCIÓN DE LA GENÉTICA EN MÉXICO:}

caso la genética - sea conocida por los científicos de un país, sino que sea practicada por ellos por medio de programas de investigación.

Pretendemos mostrar, así, que a pesar del desarrollo de ideas y posturas ante la herencia por la comunidad médica mexicana en el siglo XIX, los principios genéticos no modificaron la forma de hacer ciencia sino hasta entrados los años 40s del presente siglo a través de programas agronómicos que pretendían llevar a cabo mejoramiento vegetal y que respondían, en gran medida, a necesidades económicas derivadas de las posturas políticas de los gobiernos mexicanos.

Para mostrar lo anterior, en la primera parte analizamos las ideas que sobre la herencia se tenían en México durante las últimas décadas del siglo XIX, y cuáles eran las teorías, hipótesis o ideas que predominaban en las ciencias biológicas en México.

A pesar que que en torno a 1850 los médicos no eran el único grupo científico interesado en el estudio de los seres vivos a lo largo de la segunda mitad del siglo XIX, es esta comunidad la que más destacó por su solidez expresada en su duración en el tiempo, su temprana profesionalización e institucionalización, el número de integrantes de su organización principal, la duración de su publicación más importante y, además, la cercanía e integración de algunos de sus miembros a los grupos en el poder.

A través de la lectura de los primeros tomos de la Gaceta y de El Porvenir ${ }^{5}$ es notorio - sobre todo a partir de 1870 - , una fuerte influencia procedente tanto de la filosofía como de la medicina francesas, en la medicina mexicana. Por un lado, el positivismo de Augusto Comte introducido por el Dr. Gabino Barreda en México a finales de $1867^{6}$, y por otro, el fisiologismo de Claude Bernard, le dan un giro completo a los métodos de obtención del conocimiento utilizados hasta ese momento dentro de la medicina mexicana.

Otro cambio sensible se dejará sentir más tarde, con la introducción de la teoría evolutiva que penetra en la medicina mexicana hacia finales de la misma década de 1870.

Con ambas influencias (fisiologismo y evolucionismo) se sostiene un marco en las dos últimas décadas del siglo pasado, en el que la herencia es entendida como la transmisión del conjunto de las cualidades morales y físicas de los padres a los hijos, y se manifiesta principalmente a través de dos tendencias, comportamientos o leyes: una

\footnotetext{
5 Las publicaciones en que está basada esta investigación para este período son principalmente: la Gaceta Médica, revista de la Academia Nacional de Medicina y El Porvenir Filoiátrico, revista de la Sociedad de alumnos de la Escuela Nacional de Medicina. La Gaceta Médica comenzó a ser publicada en el año de 1864 como el «Periódico [bimensual] de la sección médica de la Comisión Científica», Tomo I: 1, 1864. Dicha Comisión fue fundada por órdenes de Maximiliano a su llegada a México en el año de 1864; la sección médica fue fundada por el Dr. Carlos Alberto Ehrmann, médico principal del ejército francés. La sección médica se convirtió después en la Sociedad Médica con el Dr. Ehrmann como primer presidente, Tomo 5:184, 1870. El Porvenir Filoiátrico fue el periódico de la Sociedad Filoiátrica y de Beneficiencia de los alumnos de la Escuela de Medicina. Tiene una duración temporal corta, su publicación comienza en 1869 y se suspende en 1874.

6 ZEA, L. (1968), El Positivismo en México, México, Fondo de Cultura Económica, p. 55.
} 
conservadora, que mantiene el tipo de la especie, y otra progresiva o acumulativa, ocasionada por los cambios ambientales y responsable de la evolución de la especie.

Posteriormente, en la segunda parte, hablaremos de la introducción de la genética en los programas de agricultura, haciendo especial énfasis en el papel que jugaron los campos experimentales de la Secretaría de Agricultura y Fomento, la Oficina de Campos Experimentales y el Instituto de Investigaciones Agrícolas.

La genética comenzó a ser utilizada por primera vez en el área de la agricultura, impulsada más bien por motivos económicos y políticos que por el desarrollo en sí de la ciencia de la genética en nuestro país.

Aunque la genética en la agricultura se conocía en México desde finales de la década de 1920, e incluso es nombrada dentro del discurso político como ciencia impulsora del desarrollo, no es sino hasta la década de 1940 cuando experimenta una gran expansión en nuestro país con el auspicio del gobierno mexicano y la Fundación Rockefeller.

Los primeros programas de investigación genética se iniciaron durante el sexenio de Lázaro Cárdenas (1934-1940) bajo la dirección del Ingeniero Agrónomo Edmundo Taboada.

La genética vegetal en México se desarrolló así, principalmente, bajo dos vertientes: la que proviene de Edmundo Taboada y la introducida por la Fundación Rockefeller. Ambas perspectivas difirieron bastante con repecto al estrato social del campesinado hacia el cual estaban dirigidas sus investigaciones, y de ahí que se enfocaran a resolver problemas distintos. Sin embargo, en cuanto a la utilización de la genética, ésta se limitó en ambos programas, principalmente a 1) la obtención de líneas puras de las variedades nativas, 2) la formación de nuevas variedades mediante hibridación, y 3) el mejoramiento mediante hibridación de las variedades creadas, de otras variedades nativas ya existentes, o de variedades importadas.

De esta forma podemos ubicar la introducción de la genética en México a través de los programas de mejoramiento vegetal.

\section{LAS IDEAS SOBRE LA HERENCIA EN LA COMUNIDAD MÉDICA MEXICANA EN LA SE- GUNDA MITAD DEL SIGLO XIX.}

Al terminar el Imperio de Maximiliano con el triunfo del movimiento reformista mexicano (1867), la comunidad médica no era desde luego, el único grupo científico relacionado con el estudio de los seres vivos ${ }^{7}$. Sin embargo, sobresale como uno de

7 Otros grupos bastante pequeños fueron el de los botánicos, los zoólogos, los agrónomos y los veterinarios. El primero contaba ya con una respetable tradición en México, pero carecía de cohesión, pues estaba constituido por unos cuantos nombres, no contaba con una academia de enseñanza propia hasta ese momento y la enseñanza de la botánica era más bien errática en los primeros 50 años del siglo XIX. 


\section{LA INTRODUCCIÓN DE LA GENÉTICA EN MÉXICO:}

los mejor conformados y establecidos del país ${ }^{8}$. Su solidez queda demostrada en ese tiempo por la conservación de su academia de enseñanza a través de los difíciles años post-indepentistas, el alto número de sus integrantes y las posiciones políticas que muchos de ellos ocupan dentro del gobierno ${ }^{9}$, en contar ya con dos revistas especializadas (La Gaceta Médica y El Porvenir Filoiátrico) y dos sociedades; en fin, por ser uno de los escasos grupos científicos que lograron mantener una tradición establecida desde la época colonial ${ }^{10}$.

A pesar de que la expulsión del ejército francés del territorio mexicano era muy reciente, en los años posteriores al Imperio se deja sentir en la medicina mexicana una profunda influencia proveniente de aquel país ${ }^{11}$.

Dicha influencia se dá principalmente a través del fisiologismo de Claude Bernard y de la filosofía de Augusto Comte.

El fisiologismo de Bernard se caracteriza por un lado, por conceder una gran importancia al medio interno o habitaculum (plasma sanguíneo) en el desenvolvimiento de todo ser vivo, debido a que es el único camino efectivo por el que el medio exterior obra en los organismos ${ }^{12}$. Por otra parte, el fisiologismo de Bernard, defiende la utilización del método inductivo (de los hechos a la teoría) y, de ahí, la observación y la utilización del experimento como las armas más indispensables para hacer una

\footnotetext{
Lo mismo puede decirse de los zoólogos, quienes conformaban un grupo todavía más pequeño. Además, son los propios médicos quienes se interesan en el estudio de ambas ramas. Así, decía el 12 de Enero de 1871 Leopoldo Río de la Loza en la Sociedad Mexicana de Historia Natural: «Hoy debemos felicitarnos... al observar que el estudio de las ciencias naturales ha salido de los estrechos y muy forzados límites a que se hallaba reducida hasta hace pocos años. Los médicos y los farmaceúticos, fueron los únicos que, obligados por las leyes concurrían poco más de una hora por unos cuantos días, a la mal organizada clase elemental de botánica, y eso sin fé en la utilidad de tal estudio, y por lo mismo, sin la dedicación indispensable, siquiera para conocer los principios fundamentales del ramo. En cuanto al de Zoología, bastará recordar que en general apenas era conocida .... a los afanes de la clase médica, auxiliada más tarde por algunas personas ilustradas y después por los ingenieros de minas, se debe la marcha progresiva y aún el entusiasmo que hoy se advierte por el estudio de las ciencias naturales». Discurso pronunciado por el $\mathrm{Sr}$. Dr. D. Leopoldo Río de la Loza, Presidente de la Sociedad Mexicana de Historia Natural en la sesión general celebrada el 12 de Enero de 1871. La Naturaleza, (1871). Tomo 1: 410. Por el lado de la agronomía y la veterinaria, la Escuela Nacional que conjuntaba ambos ramos hacía muy poco que había sido fundada (1853) y, a decir de los historiadores de las ciencias agrícolas, el estado en que se encontraba en torno a 1867 era bastante lamentable. REYES CASTAÑEDA, P. (1981), Historia de la Agricultura: información y síntesis, A.G.T. Editor. S.A, p. 120.

8 Otro grupo bastante sólido para el momento fue el de los ingenieros.

9 Un ejemplo notable es el caso del Dr. Gabino Barreda introductor del positivismo en México.

10 RodríGUEZ, J. M. (1870), «Biografía del Sr. Dr. D. Ignacio Eraza», Gaceta Médica, 5, p. 238.

11 Aquí es importante recordar que son precisamente algunos de los médicos llegados con el ejército francés, quienes fundan lo que primero fue la sección médica de la Comisión Científica que más tarde se transformó en la Sociedad Médica Mexicana.

12 ReYes, M. (1873), «Breve estudio higiénico sobre el desagüe del Valle de México», El Porvenir, 5, p. 190
} 
medicina científica ${ }^{13}$. El fisiologismo promovió una visión reduccionista de la medicina y de los fenómenos biológicos. Esta corriente adquirió vigencia en México a partir de la década de 1870.

De Comte, la medicina mexicana heredó igualmente la relevancia que Comte le concede al medio (misma que se manifiesta por ejemplo, en su definición de vida), así como consideraciones referentes al desarrollo de la ciencia ${ }^{14}$.

En cuanto a la teoría evolutiva que aparece de una manera más clara hacia finales de la década de 1870, las teorías hereditarias toman las denominadas leyes de la herencia, que se refieren principalmente a las dos tendencias en las que actúa la herencia respecto de la evolución (como es entendida por los médicos): la conservadora y la progresiva. En esta dos tendencias, sobre todo en la segunda, el medio tiene también un papel relevante.

El tema de la herencia en la medicina mexicana aparace tratado desde tres diferentes aspectos.

1) Encontrar las leyes bajo las cuales actúa.

2) Delimitar cuáles enfermedades obedecen a una causa hereditaria y cuáles obedecen a causas diferentes.

3) Conocer la causa o las causas de la alteración del germen en las enfermedades hereditarias.

El primer aspecto está en relación con un tipo de herencia que llamaremos normal, pues tiene que ver con la transmisión de caracteres no patológicos.

Los dos últimos tienen que ver con un tipo de herencia que llamaremos patológi$c a$, pues concierne a la transmisión de enfermedades.

La búsqueda de «leyes» en la medicina mexicana de las últimas décadas del siglo XIX concierne a la búsqueda de generalizaciones de tipo más bien descriptivo ${ }^{15}$.

Aparentemente, el surgimiento de la genética (1900) no tiene una repercusión inmediata en la comunidad médica. Esto, sin embargo, no significa una simple falta de

13 Introducción a la Medicina Experimental de Bernard. Citado por SEguRA, A. (1973), «La observación y la experimentación de los fenómenos biológicos». El Porvenir, 5, 221-245.

14 Para Comte, el conocimiento humano había atravesado tres períodos: el teológico, el metafísico y el positivo. Ver ZEA (1968), p. 221-222. En este trabajo el autor habla de la circunstancia mexicana y de la introducción del positivismo en México.

Para un estudio más profundo sobre el papel del positivismo en la ciencia mexicana, véase BARAHONA, A. y LeDESMA, I. (1999), «El positivismo y los Orígenes de la Biología Mexicana», Science et Techiniques en Perspective, por aparecer, y LEDESMA, I. y BARAHONA, A. (1999), «Alfonso Luis Herrera e Isaac Ochoterena: la institucionalización de la biología en México», Historia Mexicana, 48, 3, 635-675.

15 Ejemplos de estas declaraciones pueden encontrarse en el artículo de HERRERA Y FUENTES , P. (1873), El Porvenir, 5, 151-171, y LóPEZ MuÑOZ, R. (1873), «De la Fuerza Medicatriz», El Porvenir, 5, 141-150. 


\section{LA INTRODUCCIÓN DE LA GENÉTICA EN MÉXICO:}

información, sino mas bien que la «nueva ciencia» no ha sido aceptada como tal por los médicos mexicanos.

En el año de 1904, Alfonso L. Herrera habla en su libro Nociones de Biología ${ }^{16}$ de las «leyes de Mendel» (no de las leyes de la herencia) y de las «leyes de De Vries», pero consideradas no de manera independiente, sino dentro del campo de la evolución y específicamente, dentro del capítulo que denomina Hechos de la evolución; Segundo período: de demostración. Aquí, Herrera trata de una manera muy somera las «Leyes de Mendel», pues sólo se refiere a la «ley de dominancia» incluyendo algunos ejemplos en la formación de híbridos y, dado el marco evolutivo que maneja, falta por completo la teoría cromosómica de Boveri y Sutton.

En 1922, se publica la primera edición de un segundo texto de biología, Tratado Elemental de Biología, cuyo autor es otra de las figuras importantes de la biología mexicana, Isaac Ochoterena. En la $6^{a}$ edición (1942) incluye ya los capítulos sobre: Herencia (XIII); Leyes de Mendel (XIV) y Teoría para explicar la Herencia. Teoría Cromosómica (XV). En el capítulo sobre la Herencia define a ésta como «el fenómeno de la transmisión de caracteres morfológicos y de cualidades fisiológicas de un ser a su descendencia», y recalca la interacción entre el material hereditario y el ambiente, al declarar que «lo que se hereda son tendencias, es decir, nada más que potencias, posibilidades de desarrollo... la capacidad de hacer algo determinado en condiciones determinadas [...] Cada propiedad tiene una raíz doble: la constitución hereditaria y el medio ambiente» ${ }^{17}$.

Entre el año de 1900 y 1942 existe una gran distancia, y sin embargo, aunque la teoría hereditaria ha sido elaborada ya con los descubrimientos en materia de citología principalmente, la definición de herencia no varía mucho de la proporcionada por los médicos de finales del siglo XIX.

Así, al parecer, en el campo teórico la visión sobre la herencia no se modifica de manera sustancial en lss primeras décadas del siglo XX ni dentro de la comunidad médica, ni dentro de la comunidad de biólogos que se encuentra en formación.

Por otra parte, es posible que ningún programa relevante de investigación teórica en genética haya sido iniciado ni por los médicos, ni por los biólogos, sino hasta el año de 1960 con el Programa de Genética y Radiobiología, integrado en la Comisión Nacional de Energía Nuclear. Dicho programa estuvo dirigido por Alfonso León de Garay y en él actúan precisamente médicos y biólogos. Dentro de los principales objetivos del programa se encuentran diversos aspectos específicos del proceso hereditario, desde el nivel molecular hasta la Genética de Poblaciones ${ }^{18}$.

16 Herrera, A. L. (1904), Nociones de Biología, México, Imprenta de la Secretaría de Fomento.

17 Ochoterena, I. (1942), Tratado Elemental de Biología, 6a edición, México, Ediciones Botas, pp. 51-52.

18 Comisión Nacional de Energía Nuclear. Programa de Genética y Radiobiología. (1970), Informe 1960-1970, México. 
Mientras tanto, la investigación genética «práctica» o tecnológica se vino desenvolviendo con algunas décadas de anticipación en un área considerada básica para el desarrollo económico de cualquier país: la agricultura.

\section{LA INTRODUCCIÓN DE LA GENÉTICA EN MÉXICO A TRAVÉS DE LA AGRICULTURA.}

En el caso de México, durante las dos primeras décadas del siglo XX al menos, en las publicaciones médicas y en los primeros libros de texto de biología, se habla ya de «leyes de la herencia», «leyes de Mendel»e «hipótesis sobre la herencia». Sin embargo, este conocimiento no da lugar a que se inicie ningún programa de investigación sistemática en herencia o genética y así, tomando el sentido operativo de la introducción de una ciencia, no es sino hasta la década de 1930 cuando la genética realmente se introduce en México, y con una fuerza y un empuje aún mayores a partir de la década de 1940.

Quienes empiezan a planear y ejecutar programas de investigación en genética ligados a la investigación agrícola, son los agrónomos.

¿Por qué siendo los médicos una comunidad mucho más fuerte y cimentada, y siendo ellos quienes durante las últimas décadas del siglo pasado se encargaban de estudiar los fenómenos hereditarios, sobre todo desde una perpectiva reduccionista, no son ellos los primeros en hacer investigaciones genéticas? Las respuestas pueden ser diversas; sin embargo, desde nuestra perspectiva, la respuesta más acertada se encuentra más bien dentro del campo de la política y de la economía, es decir, la introducción de la genética por la vía de la agronomía, referida a las necesidades más urgentes que el país presenta en ese momento histórico, hacen que esta última reciba un apoyo mayor que otras disciplinas por parte del gobierno ${ }^{19}$.

La investigación agrícola científica coordinada por instituciones gubernamentales tiene su origen en el Porfiriato. El gobierno de Porfirio Díaz mantuvo un gran interés por fomentar la agricultura de exportación ya que ésta generaba divisas y ayudaba a

19 A las luchas armadas provocadas por la Revolución, prosiguió la lucha política por el poder en la década de 1920-1930. Entre ambas afectaron de una manera considerable a la economía mexicana en todas sus partes. Uno de los ramos que había sufrido mayores daños era el de la agricultura. Los gobiernos postrevolucionarios eran conscientes ya de la necesidad de conseguir una rápida recuperación del sector agrícola, pero el problema político y la escasez de fondos les impidió emprender las acciones necesarias. Aún en la década de 1930, con el problema político resuelto en favor del grupo de Plutarco Elías Calles, algunos agrónomos empezaron a introducir a pequeña escala los métodos modernos de genética agrícola aprendidos en universidades norteamericanas, pero, no sería hasta 1940 cuando otros factores de tipo también político y económico permitirían una utilización más expansiva de la investigación genética agrícola. 


\section{LA INTRODUCCIÓN DE LA GENÉTICA EN MÉXICO:}

equilibrar la balanza de $\operatorname{pagos}^{20}$. El apoyo para la generación de productos agrícolas de exportación incluyó no solamente las facilidades jurídicas y legales para los productores tanto nacionales como extranjeros ${ }^{21}$, sino la introducción de maquinaria e implementos agrícolas modernos, así como la aplicación de las ciencias médicas y biológicas en el cuidado de los cultivos y de los animales (principalmente ganado).

Por otra parte, la acción del gobierno de Díaz en favor de la agricultura se encaminó también a impulsar la investigación agrícola experimental y la educación agrícola superior. Gracias sobre todo a uno de los últimos Ministros de Agricultura de su gobierno, se crearon las primeras estaciones experimentales y se modernizó la Escuela Nacional de Agricultura (ENA, hoy UNACH) dando a sus carreras una orientación más técnica' ${ }^{22}$. Así, 1907 el nuevo programa de la Escuela Nacional de Agricultura incluía la formación de Ingeniero Agrónomo, Médico Veterinario y de técnicos en Mecánica Agrícola y en Agronomía ${ }^{23}$.

Al mismo tiempo, se planeó el establecimiento y operación de una estación experimental de investigación anexa a la Escuela de Agricultura. Dicha estación sería la estación central y coordinadora de otras tantas que estarían ubicadas en Cd. Juárez, Chih. ${ }^{24}$, Río Verde, S.L.P., Villahermosa, Tab., Oaxaca, Oax., El Dorado, Sin., Sn. Pedro, Coah. y una en Yucatán. El Ministerio de Agricultura contrató especialistas de Francia, pero con el inicio de la revolución en 1910, sólo llegaron a operar las primeras cuatro ${ }^{25}$.

20 Webster, G, von. (1992), «La Agricultura en el Porfiriato: 1876-1911», Primer Simposio de ciencias agronómicas, México, Colegio de Postgraduados en Ciencias Agrícolas de Chapingo, pp. 135-137.

21 Por ejemplo, para la adquisición de predios «baldíos» (que en muchos de los casos pertenecían a comunidades indígenas) y el uso de aguas federales.

22 Por iniciativa del Ministro Olegario Molina, el Congreso de la Unión expidió el decreto de 14 de Noviembre de 1907 para que la Escuela Nacional de Agricultura pasara nuevamente a depender de la Secretaría de Fomento. En la exposición de motivos pide que ésta deje de ser dependencia de la Secretaría de Instrucción Pública y argumenta la importancia que la agricultura ha ido adquiriendo para la nación, el «progreso» que ha alcanzado y lo poco que éste corresponde al nivel en que se encuentra la educación agrícola superior. Así, al depender la Escuela de Agricultura directamente de la Sría. de Fomento, se podrá vigilar más de cerca lo que en ella se enseña y de esta manera, la formación de los técnicos que el país necesita. Tomado de EsCOBAR, R. (1909), La Instrucción Agrícola en México, México, Secretaría de Agricultura y Fomento, pp. 49-56.

23 REYES, P. (1981), Historia de la Agricultura: información y síntesis, México, A.G.T., Editor. S. A., p. 127.

24 La estación experimental de Cd. Juárez estaba anexa a una escuela de agricultura fundada en ese estado en 1906 por el Ing. agrónomo Rómulo Escobar. Aunque la escuela era particular, la investigación llevada a cabo en el campo experimental era publicada por la Secretaría de Fomento.

25 VAllejo, J. R. (1992), Historia de la Agricultura y de la Fitopatología (con referencia especial a México), Colegio de Postgraduados en Ciencias Agrícolas, Chapingo, México, p. 67. 
En las estaciones experimentales se produjo una considerable cantidad de publicaciones - al menos 45 circulares y 80 boletines- a pesar de los pocos años durante los que funcionaron (de 1908 a 1914 aproximadamente) ${ }^{26}$.

\subsection{La investigación agrícola y la utilización de la genética en el período 1920-1960.}

En La modernización de la agricultura mexicana: 1940-196027, la politóloga Cynthia Hewitt de Alcántara habla acerca de las consecuencias sociales que tuvo la introducción de la «revolución verde» en México, en la calidad de vida del campesinado.

Según Hewitt, a finales de las décadas de los 30's y los 40's había dos corrientes políticas dentro de los círculos de poder encargados de guiar y dirigir las acciones a seguir en el agro mexicano, que se reflejaron e influyeron en las instituciones encargadas de la investigación en genética vegetal. Esta división en el poder político del país tuvo sus raíces en el Porfiriato, y se reflejó en el movimiento de la Revolución Mexicana como una lucha de clases. Así, existían quienes luchaban por la creación de una agricultura campesina viable basada en las tradiciones de tenencia comunal de la tierra anteriores a la revolución, y los que propugnaban por la empresa privada en gran escala. Era en el fondo, en palabras de Cynthia Hewitt, «un conflicto entre los intereses del campesinado y los de una clase media en marcha, entre los partidarios de Zapata y los de Carranza» ${ }^{28}$.

Aunque la atención al mejoramiento de la tecnología agrícola formó parte intermitente de la política oficial en México desde que empezó el siglo, la investigación destinada a aumentar la producción de alimentos para el consumo nacional (recuérdese que a finales del Porfiriato se fomentaban los cultivos de exportación) no empezó a escala digna de mención sino en los años treinta, en especial en el período de la administración de Lázaro Cárdenas del Río (1934-1940), y con mayor fuerza en los 40's.

La filosofía de desarrollo económico de Cárdenas era agrarista, estaba en línea con el pensamiento de Zapata; Cárdenas creía que la mayor productividad del campo mexicano estaba íntimamente vinculada a cambios de estructura que transformarían las grandes propiedades agrícolas capitalistas en cooperativas de campesinos y jornaleros. De esta manera, los primeros agrónomos mexicanos formados en la aplicación de las nuevas tecnologías agrícolas compartían la misma filosofía de Cárdenas y se

26 La temática de las publicaciones es variada, pero predominan principalmente los tópicos relacionados con las enfermedades y plagas de plantas y animales, y los temas en veterinaria general, así como las instrucciones sobre cómo sembrar adecuadamente ciertos cultivos (como la cebada, el lúpulo, la higuera, el arroz, etc.), o recomendaciones sobre la conveniencia de introducir otros nuevos.

27 HewITT, C. (1985), La modernización de la agricultura mexicana: 1940-1970. Siglo XXI editores, $5^{\mathrm{a}}$ edición, México.

28 HewitT (1985), p. 17. 


\section{LA INTRODUCCIÓN DE LA GENÉTICA EN MÉXICO:}

preocupaban más por hallar soluciones a los problemas de índole práctica que enfrentaban el común de los campesinos, que por importar tecnología extranjera ${ }^{29}$.

El grupo cardenista, encabezado por el Ing. Agrónomo Edmundo Taboada Ramírez, se formó y llevó a cabo sus investigaciones dentro de los campos experimentales de la Secretaría de Agricultura fundados en 1933. El conjunto de campos experimentales se convirtió más tarde en el Departamento de Campos Experimentales (DCE), y hacia finales de los cuarenta, en el Instituto de Investigaciones Agrícolas (IIA).

Por otro lado, al comenzar la década de 1940 y al cambio de gobierno de Cárdenas por Manuel Ávila Camacho (1940-1946), resurgió en el poder la tendencia capitalista en la agricultura; la tendencia a incrementar la producción en el próspero sector privado de la agricultura mexicana de manera que pudiera proveer de un excedente que permitiera alimentar a las ciudades en rápida expansión y aprovisionara a las nuevas industrias ${ }^{30}$.

Dentro de la tendencia capitalista se formó otro grupo de investigadores mexicanos en agricultura integrados en el llamado Programa Agrícola Mexicano (específicamente dentro de la Oficina de Estudios Especiales) de cooperación entre el gobierno mexicano y la Fundación Rockefeller de Estados Unidos. Es decir, integrados en la introducción del «paquete tecnológico» característico de la «revolución verde» que empezó en México y fue transferida más tarde a otros países del Tercer Mundo.

A pesar de que en el fondo estas dos tendencias delineadas por Hewitt tienen los mismos objetivos - lograr un aumento en la producción de alimentos básicos en México- y que, como veremos, emplearon la misma metodología, según la autora, además del tipo de productor agrícola hacia quién y para quién estas dos líneas enfocaban la investigación agrícola, existió entre ambas una importante diferencia en cuanto al apoyo económico y administrativo brindado por el gobierno federal durante los 40's y 50's. Las discrepancias de tipo político propiciaron a su vez una relación más bien distante entre ambas instituciones durante esas décadas.

La primera tendencia, la agrarista, es la que conduce al Instituto de Investigaciones Agrícolas y su figura principal es Edmundo Taboada Ramírez. La segunda, corresponde a la mirada capitalista, y nos lleva a la Oficina de Estudios Especiales, dirigida a lo largo de toda su existencia por investigadores norteamericanos. Ambas líneas se fundieron finalmente en 1960 para formar el Instituto Nacional de Investigaciones Agrícolas (INIA) cuya trayectoria ya no es tratada en este trabajo por tratarse de una segunda etapa en la historia de la agronomía en México.

\footnotetext{
29 HewitT (1985), p. 31.

30 HewitT (1985), p. 32.
} 
2.1.1. Los campos experimentales de la Secretaría de Agricultura y Fomento (SAF), la Oficina de Campos Experimentales y el Instituto de Investigaciones Agrícolas (IIA).

Desde mediados de la década de 1920, en los expedientes de agricultura de la Secretaría de la Presidencia se habla del «mejoramiento vegetal» de cultivos como el algodón, ${ }^{31} \mathrm{y}$ «del estudio de las variedades cultivadas, de la conveniencia de introducir otras nuevas o mejorar las que existen» y de la «catalogación de los híbridos y sus posibilidades» para el caso del olivo ${ }^{32}$.

Hasta el año de 1933 en que se crean algunos campos experimentales dentro de dicha Secretaría, la investigación en genética vegetal no encuentra un terreno más o menos propicio para la continuidad ${ }^{33}$. El Ing. agrónomo Edmundo Taboada Ramírez es la persona que se encarga de ello y es, de hecho, el personaje principal en los años subsecuentes dentro de esta línea.

Edmundo Taboada, egresado de la Escuela Nacional de Agricultura de Chapingo, fue «el primer técnico mexicano en agronomía que tuvo la oportunidad de hacer estudios de postgrado durante 1932 y 1933 en la Universidad de Cornell, N. Y., sobre genética vegetal, y en la de Minessota estudios con el Dr. E. Stackman en parasitología vegetal», ${ }^{34}$ específicamente en el chahuixtle del trigo. Fue uno de los primeros catedráticos de genética vegetal y aplicada en la Escuela Nacional de Agricultura, y el primer autor nacional en escribir un libro de texto sobre genética general -Apuntes de Genética- ${ }^{35}$.

Al comenzar el período administrativo de Lázaro Cárdenas (1934), Taboada fue designado Jefe de la estación agrícola experimental del Yaqui en Sonora, creada el año anterior en el Departamento de Agronomía, Química y Suelos de la Dirección General de Agricultura de la SAF. En esta estación Taboada inició el primer trabajo en genética vegetal, el cual consistió en seleccionar de entre diversas variedades de ajonjolí la que estuviera mejor adaptada a las condiciones ecológicas del Valle del Yaqui. Las siguientes selecciones que llevó a cabo dentro del distrito de riego de Pabellón, Aguascalientes, en los años de 1936 y 1937 dieron como resultado la ob-

31 Secretaría de la Presidencia Expediente 104-C-15.

32 Secretaría de la Presidencia, Expediente 241-A-O-13.

33 De cualquier manera, la investigación que se empezó a realizar en los campos experimentales a partir de 1933 tuvo un carácter local, pues los resultados no fueron difundidos al resto del país y las selecciones hechas en las variedades sólo funcionaban para esa región. Por otra parte, la investigación agrícola sufrió los altibajos administrativos de una serie de cambios que se dieron dentro de la SAF durante la década de 1930. Estas alteraciones en su estructura organizativa iban y venían con las diferentes administraciones de gobierno (se pueden consultar los informes de actividades que anualmente presentaba dicha institución ante el Congreso de la Unión a partir del año de 1934).

34 INIA. (1985), Edmundo L. Taboada Ramírez: una semblanza, 1906-1983. SARH, INIA, Publicación especial. México, p. 7.

35 Taboada, E. (1938), Apuntes de Genética, Escuela Nacional de Agricultura, Chapingo, México. 


\section{LA INTRODUCCIÓN DE LA GENÉTICA EN MÉXICO:}

tención de una variedad de chile pasilla de mayor rendimiento y mejores características agrónomicas que las otras variedades regionales ${ }^{36}$.

En 1940, en la dirección General de Agricultura se creó la primera oficina de experimentación agrícola del país, la Oficina de Campos Experimentales y su primer Jefe fue precisamente Edmundo Taboada. Los primeros diez campos experimentales coordinados a nivel nacional fueron: El Yaqui, Son., Llera, Tamps., Pabellón, Ags., Briseñas, Mich., León, Gto., Querétaro, Qro., Tlalnepantla, Mex., Zacatepec, Mor., El Palmar, Ver., y Rosario Izapa, Chis ${ }^{37}$.

En sus primeros seis años de vida, en estas estaciones experimentales se seleccionaron diferentes variedades de maíz adaptadas a las condiciones ecológicas y económicas de los productores de diferentes estados del país: la variedad Celaya, Guanajuato 16 y Guanajuato 21 para el estado de Guanajuato; la Jalisco 35 para el estado del mismo nombre; la Chapingo 1 y la Hidalgo 7 para el Valle de México que tenía bajo riego y la Llera III para Tamaulipas, maíz precoz y con buen rendimiento propicio para las zonas bajas del noroeste del país ${ }^{38}$. Estas variedades de maíz llamadas «de polinización abierta» resultaban particularmente adecuadas para la mayoría de los productores de maíz, pues como explicaba Taboada en el informe presentado a la Secretaría de Agricultura (SAG) en el período 1951-1952:

«Hay varios tipos de semillas de maíz de alto rendimiento. Los rendimientos mayores se obtienen con los maíces llamados 'híbridos', pero su excepcional productividad sólo dura para la primera siembra. En las siembras siguientes, la productividad baja tan señaladamente que a veces los rendimientos son inferiores a los que puede obtenerse con semillas ordinarias, obligando así al agricultor a adquirir semilla nueva cada año. Gran número de los que cultivan el maíz en México no pueden emplear con éxito estos maíces híbridos, sea por sus limitados recursos económicos, sea por sus limitados conocimientos.

Las variedades mejoradas de polinización abierta son otro tipo de maíz de alto rendimiento. Aunque a veces son algo menos productivas que las híbridas, tienen la gran ventaja de la permanencia, y el agricultor puede destinar una parte de su cosecha para semilla del año siguiente exactamente como suelen hacer nuestros pequeños agricultores cuando siembran $»^{39}$

En el cultivo del trigo se iniciaron las colecciones de variedades en los campos de los agricultores, se realizaron ensayos de rendimiento de aquéllas que tenían las mejores características agronómicas y se empezaron a hacer los primeros cruzamientos entre las variedades mexicanas con alto rendimiento, pero susceptibles al chahuixtle del tallo y las variedades americanas con escasa adaptación pero resistentes a esa

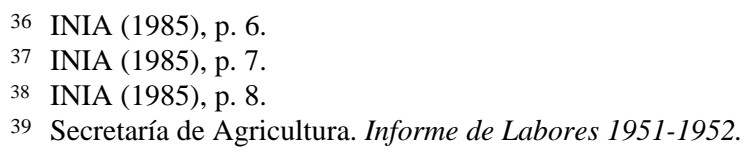


enfermedad. Posteriormente se distribuyeron las variedades Lagunero y Anáhuac para el norte del país ${ }^{40}$.

En arroz se iniciaron las selecciones y se sembraron los primeros ensayos de rendimiento de las variedades Jojutla y Colima que en años posteriores fueron distribuídos para producción comercial.

En otros cultivos de importancia comercial, como el ajonjolí, se empezó a trabajar en los años de 1944-1946 en la creación de variedades por medio de selección individual y cruzamiento. Dados los problemas ocasionados en la industria de la producción de llantas y otros productos del hule durante la Segunda Guerra Mundial, se inició en 1942 un programa de experimentación con clones importados de Sumatra, Java, Malaya y Filipinas; a finales de 1945 ya se estaban estableciendo las primeras plantaciones en la región de El Palmar, Veracruz. En los años de 1944-1945 se hizo una prueba de introducción de 120 variedades de caña de azúcar. En 1945 también, se empezó a construir la Estación Central de Investigación y Propagación del Cacao en Rosario Izapa, Chiapas, en la cual se probaron clones provenientes del Colegio Imperial de Agricultura de la Isla De Trinidad; sin embargo, el programa más importante fue el de selección de clones nativos cuya fase inicial consistió en la colección de frutos de más de 65.000 árboles localizados en diferentes plantaciones del estado de Chiapas ${ }^{41}$.

Otros programas de investigación fueron hechos en cultivos de frijol, papa, algodón, olivo, higuerilla, cáñamo y guayule.

En 1947 la Oficina de Campos Experimentales se transformó en el Instituto de Investigaciones Agrícolas (IIA) con Edmundo Taboada como Director hasta el año de 1960. Durante ese período (1947-1960) se realizaron una gran cantidad de programas de investigación en muy diversos cultivos; en realidad hubo una continuidad de los programas iniciados por la Oficina de Campos Experimentales y los del IIA ya que tenían básicamente el mismo cuerpo técnico, el cual se incrementó sustancialmente con el tiempo así como el número de campos experimentales. ${ }^{42}$ En el maíz se crearon, además de las ya mencionadas, las variedades Costeño 52 para la costa de Culiacán, Sinaloa, los híbridos H-52 y H-58 para otras zonas tropicales y la Llera339 para terrenos altos de temporal.

Edmundo Taboada personalmente se dedicó a producir lo que llamó variedades estabilizadas de maíz tomando como base las variedades de maíces de polinización abierta creadas durante los años anteriores. El método básico para producirlas consistía, primero, en la obtención de líneas con los menores defectos agronómicos y con buena aptitud combinatoria. Luego, por medio de cruce $\mathrm{AB}$ y su evaluación posterior, se seleccionaban aquellas con mayor rendimiento. Después se realizaban todas

40 INIA (1985), p. 8.

41 INIA (1985), pp. 9-10.

42 INIA (1985), p. 10. 


\section{LA INTRODUCCIÓN DE LA GENÉTICA EN MÉXICO:}

las combinaciones posibles entre las líneas superiores produciendo así una población con equilibrio genético. Con esta metodología se obtuvieron en la década de los cincuenta numerosas variedades estabilizadas que fueron distribuídas entre los agricultores de diferentes regiones del país. ${ }^{43}$

En 1960, el IIA se fusionó con la otra dependencia que desde el año de 1944 comenzó a hacer de manera paralela investigación agrícola en México: la Oficina de Estudios Especiales perteneciente al Programa Agrícola Mexicano.

\subsubsection{El Programa Agrícola Mexicano y la Oficina de Estudios Especiales (OEE): la «revolución verde»}

En 1941, unos pocos meses después de que Ávila Camacho remplazara a Cárdenas en la presidencia, el gobierno de México inició conversaciones informales con unos representantes de la Fundación Rockefeller sobre la posibilidad de un programa de ayuda técnica, en virtud del cual el personal proporcionado por la fundación podría laborar en la superación de la productividad agrícola en México. Ese mismo año la fundación envió un comité de tres especialistas en agricultura para que hicieran un viaje de reconocimiento e informaran sobre la conveniencia y posibilidades de iniciar un programa patrocinado por la misma. Este comité estaba integrado por el Dr. E. C. Stakman, Jefe de la División de Fitopatología de la Universidad de Minesotta, el Dr. Paul Mangelsdorf, Director del Museo Botánico de la Universidad de Harvard y el Dr. Richard Bradfield, Jefe del Departamento de Agronomía de la Universidad de Cornell. El comité visitó más de la mitad de los 28 estados de la República investigando los cultivos característicos de las diversas regiones; los tipos de climas, suelos, tradiciones y costumbres de su gente. El informe presentado por este comité fue favorable y casi en seguida el Gobierno de México hizo una invitación formal a la fundación para llevar adelante un programa cooperativo de agricultura en México. La fundación aceptó la invitación y el Programa Agrícola Mexicano se inició en febrero de $1943^{44}$.

Después de iniciado el programa oficialmente, el Dr. Stakman regresó a México con el Director del programa, J. G. Harrar, para consultar con los científicos mexicanos y establecer las bases y las acciones específicas a seguir. Ambos doctores procedieron de acuerdo con el informe preeliminar del comité consultivo, que recomendaba la concentración inicial en las siguientes ramas de la ciencia agrícola: 1) suelos, 2) genética, 3) control de las enfermedades y plagas y 4) ganadería. Los especialistas americanos y los investigadores mexicanos acordaron tras hacer un estudio exhausti-

43 INIA (1985), p. 10.

44 Harrar, J. G. (1950), Programa Agrícola Mexicano, Fundación Rockefeller (1913), Leo Hart Co., Nueva York, U.S.A, pp. 5-6. 
vo, un programa de doble fin: la actividad central sería la investigación fundamental de métodos y materiales de utilidad para incrementar los cultivos básicos alimenticios pero, como este programa con el tiempo debería ser totalmente mexicano, se acordó que se diera mayor énfasis en segundo lugar a un programa de adiestramiento para investigadores mexicanos seleccionados ${ }^{45}$.

Con estas bases se creó en 1944 la Oficina de Estudios Especiales (OEE) cuyas oficinas junto con los laboratorios auxiliares de fitopatología y entomología quedaron ubicados en el edificio de la Dirección General de Agricultura en Sn. Jacinto, ciudad de México. La estación experimental quedó situada en terrenos de la Escuela Nacional de Agricultura de Chapingo y en ella se establecieron algunos laboratorios de campo, invernaderos y proyectos científicos de siembra. Posteriormente el Programa inauguró otra estación experimental en Guanajuato, y una más en Morelos. El personal de la estación de Chapingo quedó compuesto por especialistas en todas las fases de la ciencia agrícola; químicos de suelos, genetistas, microbiólogos, fitopatólogos, entomólogos, ecólogos e ingenieros agrícolas ${ }^{46}$.

Como los dos cereales con una mayor superficie cultivada en el período 19391941 ( $72 \%$ de la superficie total del país $)^{47}$ y como parte primordial de la dieta de los mexicanos (sobre todo el primero), el eje del programa de investigación de la OEE fue el mejoramiento del maíz y del trigo.

La experimentación comenzó por formar un banco de información genética con semillas provenientes de muchas regiones productoras de la República, procurando que quedaran incluídas todas las regiones. Los investigadores llevaron semillas de más de mil variedades de maíces nativos a la estación experimental de Chapingo. Ahí, y en parcelas experimentales distribuídas en todo el país, sembraron las semillas junto con otras provenientes de Estados Unidos, Guatemala, y otros países productores de maíz con el fin de averiguar cuáles tenían las mejores características agronómicas, como mayor rendimiento, precocidad, valor nutritivo y adaptación climática. Este ensayo preliminar les dió una base para seleccionar unas cuantas variedades buenas para la multiplicación de su semilla. Las primeras variedades así obtenidas fueron entregadas para su multiplicación y se destinaron para su uso inmediato distribuyéndolas rápidamente a los agricultores para su siembra. Luego, los investigadores comenzaron a hacer nuevo cruce y desarrollaron nuevas variedades sintéticas e híbridas. El proyecto del mejoramiento del maíz de la OEE dió origen al establecimiento de una de las colecciones más grandes y más variadas de maíz en aquel tiempo ${ }^{48}$.

45 HARRAR (1950), p. 14.

46 HARRAR (1950), pp. 15-16.

47 Centro de Investigaciones Agrarias. (1974), Estructura agraria y desarrollo agrícola en México, México, p. 174.

48 HARRAR (1950), pp. 25-26. 


\section{LA INTRODUCCIÓN DE LA GENÉTICA EN MÉXICO:}

En el trigo, uno de los problemas más grandes era el la roya o chahuixtle que en años favorables para este hongo devastaba los cultivos ocasionando grandes pérdidas. Norman Bourlag, jefe del programa de experimentación triguera de la OEE, encontró que en México existía la siguiente situación al inicio del programa en 1944:

\footnotetext{
El trigo se cultivaba primordialmente en invierno y en regadío. Se sembraba de septiembre a diciembre y se cosechaba de abril a junio, según las variedades y la altura. Las variedades eran todas de primavera y de origen desconocido, menos dos de ellas, la Ramona y la Baart, que procedentes de California habían sido introducidas en el estado de Sonora. No había variedades en el sentido corriente de la palabra, sino mezcla de muchos tipos diferentes. Todas las variedades eran susceptibles al chahuixtle o roya del tallo y de las hojas. En los años en que las condiciones ecológicas eran favorables al moho patógeno del tallo, como fue el caso en Sonora en 1939, 1940 y 1941 y en la región central del Bajío en 1948, devastadoras epidemias llevaron a la ruina a los cultivadores de trigo. Las prácticas [de cultivo] eran primitivas en todas las regiones menos en Sonora, que estaba mecanizada. En todas las demás partes, el único instrumento empleado en la preparación de la tierra y las operaciones de siembra era el antiguo arado de madera, tirado por bueyes o mulas. En todas partes menos en Sonora, se cosechaba con una hoz y la trilla se efectuaba con pequeñas trilladoras fijas o más comúnmente se hacía salir el grano pisando las espigas los bueyes o las mulas, y zarandeándolo después para limpiarlo. Los rendimientos eran bajos y estancados, con un promedio nacional de $750 \mathrm{~kg}$ por hectárea ${ }^{49}$.
}

Se puso en práctica entonces, primero mediante la selección de las semillas nativas e importadas de máximo rendimiento para su distribución inmediata a los agricultores comerciales, y después, progresivamente, cruzando las mejores variedades para formar nuevas combinaciones genéticas.

Como parte del programa de selección, unos representantes de la OEE fueron enviados al campo y a estaciones experimentales de regiones trigueras para recoger muestras de semilla e interesar a los agricultores locales o a los empleados de gobierno para participar en el programa permitiendo que se pusieran a prueba pequeñas cantidades de semilla experimental en sus tierras. En total, se reunieron 11.000 plantas indígenas diferentes y se trajeron del extranjero unas cuatrocientas variedades para mayor comprobación. Cuatro de estas últimas se estaban multiplicando en 1947 para su utilización comercial (denominadas Rocamex 211, 209, 321 y 324, procedentes de Kenia y Texas) y al año siguiente una quinta, Gabo, procedente de Australia ${ }^{50}$.

A partir de 1945, el programa de selección se desarrolló junto con esfuerzos sistemáticos para cruzar las mejores variedades locales con las extranjeras, y producir variedades enteramente nuevas adaptadas especialmente a México. La metodología utilizada para producir las nuevas variedades consistía en:

\footnotetext{
49 HewitT (1985), p. 37.

50 HewitT (1985), pp. 38-39.
} 


\section{ANA LILIA GAONA ROBLES y ANA BARAHONA ECHEVERRÍA}

El investigador recurre a la hibridación. Escoge como padres dos variedades con las características que desea combinar [...]. Para cruzarlas, primero emascula una de ellas quitándole las anteras, los órganos masculinos que contienen el polen, con delicados fórceps cuando esos órganos están bien desarrollados pero todavía no maduros. Después cubre la cabeza emasculada con una bolsita de papel cristal para impedir la polinización no controlada. A los pocos días cuando los órganos femeninos, los estigmas, se han hecho receptivos, el operador los poliniza con anteras maduras de la otra variedad.

Esta polinización produce semillas que forman plantas híbridas de primera generación, perfectamente uniformes, y nada se consigue practicando la selección sobre ellas. Pero en la segunda generación y las que siguen, la segregación genética crea nuevas combinaciones tan abundantes y diversas como las cartas de una baraja. Las oportunidades de selección creativa son enormes. Es en las primeras generaciones que siguen a una cruza cuando el fitocultor muestra su talento, porque es entonces cuando tiene que seleccionar para su propagación las combinaciones que más se aproximan al trigo ideal que se ha propuesto crear ${ }^{51}$.

Después de nueve años, este programa produjo y entregó a los agricultores trece nuevas variedades comerciales de trigo que satisfacían todos los requisitos de rendimiento y resistencia al chahuixtle establecidos por la OEE (Yaqui, 48, 50 y 53; Mayo 48 y 54; Kentana 48; Chapingo 52 y 53; Bajío 53; Toluca 53; Mexe 53 y Sinaloa 54).

Así, a comienzos de la década del cincuenta, estas primeras variedades de alto rendimiento llegaron a ser empleadas en una gran parte de las tierras trigueras del país. No obstante, la guerra contra el chahuixtle estaba lejos de haber terminado porque las especies de chahuixtle que llevaban veinte años o más inactivas reaparecieron súbitamente, y otras más recientes que se suponía vencidas por la investigación genética dieron muestras de una notable capacidad para adaptarse a las nuevas condiciones. Entre 1951 y 1954, cinco virulentas especies del parásito reaparecieron, atacaron a algunas de las variedades nuevas de alto rendimiento e hicieron necesario que el programa de la OEE se pensara en función de la creación de un nuevo trigo compuesto de una mezcla de diversas variedades que conservara las características productivas de las anteriores variedades, pero que además fuera resistente a diferentes variedades de chahuixtle. Por fortuna, la espectacular resistencia de la variedad Lerma Rojo, entregada a los agricultores en 1954, hizo innecesario seguir adelante con un plan tan complicado ${ }^{52}$.

La característica a la que se dió particular importancia en las investigaciones de la OEE en años siguientes, aparte de la resistencia al chahuixtle, fue la capacidad de responder las nuevas variedades a un «paquete» cada vez más complejo de insumos y prácticas, como fertilizantes químicos, herbicidas, insecticidas, irrigación y mecanización, todos especialmente aplicados a las zonas de riego.

La culminación del esfuerzo para crear semillas que aumentaran el rendimiento y resistieran al chahuixtle se presentó en 1961-1962 cuando se entregaron a los agricultores mexicanos los famosos trigos enanos. Estas variedades de tallo corto se habían

\footnotetext{
51 HEWITT (1985), p. 39.

52 HewitT (1985), pp. 42-43.
} 


\section{LA INTRODUCCIÓN DE LA GENÉTICA EN MÉXICO:}

hecho necesarias precisamente por la incapacidad de los trigos anteriores de alto rendimiento, incluso el Lerma Rojo, para aguantar grandes dosis de fertilizante sin que se «encamaran» (abatieran) antes de que fuera el tiempo de la cosecha. Las barreras impuestas por el encamado habían sido reconocidas ya por el personal de la OEE en 1948, pero no se había encontrado forma utilizable de enanismo hasta el descubrimiento del trigo japonés Norin, incorporado al programa triguero mexicano en 1953. Entre ese año y 1962 se perfecccionaron nuevas variedades de tallo corto (como la Pénjamo, Pitic y la Sonora 63 y 64) que aumentaron mucho la productividad de los trigales mexicanos, más aún que la Lerma Rojo ${ }^{53}$.

Otras investigaciones desarrolladas por la OEE de manera secundaria a las investigaciones con el maíz y el trigo, se hicieron en 1949 con frijol, y dieron como resultado la selección de 7 de sus variedades recomendables por su rendimiento superior y su resistencia a las enfermedades y plagas. En 1952 con papa, en 1953 con algunas hortalizas y verduras, en 1954 con sorgo, cebada y leguminosas forrajeras, y en 1956 con ganado ${ }^{54}$.

Por otra parte, en el programa de adiestramiento para investigadores mexicanos, la Secretaría de Agricultura y Ganadería (SAG) comisionaba a un grupo de 15 ó 16 jóvenes sobresalientes graduados de las principales escuelas de agricultura de México, asignándolos a la Oficina de Estudios Especiales. Estos jóvenes agrónomos participaban directamente en los trabajos de campo y de laboratorio durante un año o un poco más, y después eran envíados — según sus aptitudes para la investigaciónpara hacer estudios en el extranjero (principalmente a Estados Unidos). Los estudiantes mexicanos hacían estancias de un año cuando menos, becados en la institución más adecuada a las necesidades de su especialidad; al finalizar debían regresar a México a ocupar puestos como investigadores agrícolas al servicio del gobierno.

En la OEE se aceptaban también estudiantes de otros países de Latinoamerica, lo cual permitió que en el año de 1949 se pudiera celebrar una Primera Asamblea Latinoamericana de Fitogenetistas. Esta Asamblea tuvo lugar en la ciudad de México, siendo anfitrona la OEE, y en ella presentaron numerosos trabajos los investigadores mexicanos que laboraban en dicha Oficina ${ }^{55}$

El programa de adiestramiento de la OEE tuvo un buen resultado, pues a lo largo de la década de 1950 un número cada vez mayor de mexicanos iban ocupando los puesto importantes (aunque los puestos directivos siempre fueron ocupados por norteamericanos). Así por ejemplo, en 1956 la OEE contaba con 18 especialistas de tiempo completo de la fundación y con más de 100 científicos mexicanos. Finalmente, cuando la Rockefeller se retiró del Programa Agrícola Mexicano en 1960 y la OEE se fusionó con el IIA, se habían formado una gran cantidad de investigadores mexicanos.

\footnotetext{
53 HewitT (1985), pp. 44-45.

54 HARRAR (1950), pp. 28-29.

55 Oficina de Estudios Especiales (1959), Primera Asamblea Latinoamericana de Fitogenetistas. Secretaría de Agricultura y Ganadería, México.
} 


\subsubsection{Comparación metodológica entre las dos líneas de investigación en genética agrícola. ¿Qué tipo de genética se introdujo en México?}

Independientemente de las tendencias políticas y del nivel económico y social de los productores agrícolas hacia quienes estaban orientados los resultados positivos de la investigación en genética agrícola realizadas por la línea de Edmundo Taboada y del Programa Agrícola Mexicano, metodológicamente, ambas líneas seguían un patrón común en la investigación. Es decir, ésta siempre comenzaba por reunir una colección de material genético formado por las semillas de la planta sujeto de experimentación (maíz, trigo, frijol, etc.) procedentes de diversas regiones de la República y de algunas extranjeras, de su siembra en campos experimentales y de la observación de las características de interés fitotécnico que poseía cada una de ellas, seguido de una selección de aquellas variedades que presentaran las características más adecuadas al objetivo perseguido (mayor rendimiento, mayor resistencia a las enfermedades, precocidad, etc.). Una vez obtenidas estas variedades se podía continuar con la experimentación haciendo cruce entre variedades con el fin de producir variedades híbridas con características aún mejores que las variedades parentales.

Esta metodología, es en efecto, la misma utilizada por los fitotecnistas norteamericanos desde principios de siglo.

La genética aplicada al mejoramiento vegetal o fitotecnia comenzó a ser utilizada casi inmediatamente después del «redescubrimiento» de las leyes de Mendel por E. M. East y C. H. Shull en la Estación Experimental Agrícola de Connecticut y en Cold Spring Harbor respectivamente en el año de $1905^{56}$. Estos primeros estudios de endocría y cruzamiento fueron hechos en el maíz. A partir de entonces este tipo de investigación se extendió a otras universidades de agricultura de los Estados Unidos como la de Minnessota, California, Washington y Ohio, Ilinois entre otras. En un principio, los resultados alcanzados por la fitotecnia norteamericana no lograron atraer el interés de los agricultores norteamericanos, pero en torno a la década de 1930, conforme iba quedando demostrada la gran diferencia existente entre las variedades normalmente utilizadas y las producidas por la experimentación (por ejemplo, con respecto de la resistencia a las enfermedades), esta disciplina se volvió muy popular entre el gran público que se fue mostrando cada vez más dispuesto a cooperar con las investigaciones e incluso a financiarlas.

Es precisamente este tipo de genética la que se introduce en México a mediados de la década de 1930, y sobre todo en la década de 1940: la genética aplicada al mejoramiento vegetal.

Históricamente, desde principios de siglo se desarrolló en Estados Unidos una escuela fuerte de genética clásica ligada a la agricultura, la llamada genética del maíz,

\footnotetext{
56 HAYES, K. H. (1947), Métodos fitotécnicos; procedimientos científicos para mejorar las plantas cultivadas, Ediciones ACME, Bueno Aires, Argentina.
} 


\section{LA INTRODUCCIÓN DE LA GENÉTICA EN MÉXICO:}

cuyo pionero y cabeza fue R. Emerson de la Universidad de Cornell. Los investigadores de la genética del maíz se dedicaron durante las tres primeras décadas de este siglo a determinar el mecanismo de la herencia mendeliana que estaba siendo desarrollada por T. H. Morgan y sus colaboradores, primero en Columbia y luego en el Tecnológico de California. Uno de los principales objetivos era el de hacer un mapeo de los cromosomas del maíz. ${ }^{57}$ Este grupo de investigación estuvo íntimamente ligado a los programas de desarrollo agrícola.

La genética clásica del maíz ligada al mejoramiento vegetal no se practicó en México en ningún momento. Sin embargo, los investigadores mexicanos que se formaron en fitotecnia, poseían el cuerpo completo de los conocimientos genéticos básicos necesarios para comprender cuáles eran los mecanismos hereditarios que actuaban durante las experimentaciones (como lo prueban los Apuntes de Genética de 1938 escritos por E. Taboada). Y por otra parte, un considerable entendimiento de la genética de poblaciones que a su vez se desarrollaba de manera independiente de la genética del maíz.

Es por tanto el lado práctico de la genética, aquel ligado a la agricultura, el que primero se introduce en México.

\section{CONCLUSIONES}

El desarrollo de la genética en México tiene un paralelo con el desarrollo de esta ciencia en Europa y Estados Unidos sólo en algunas de sus vertientes. Es decir que, para la etapa en que no se habla todavía de genética (antes de 1900), sino de un concepto más amplio, el de herencia, en México este concepto es manejado y conceptualizado principalmente dentro de la medicina. Posteriormente a 1900, cuando surge la genética como ciencia, en México se desarrolla por su lado más práctico o tecnológico: el mejoramiento de especies.

Las áreas en las que se desarrolla el concepto de herencia y la genética en México, generalmente no son incluídas en las historias tradicionales de la genética.

Entonces, para hacer un análisis de las verdaderas contribuciones de las comunidades de médicos y de agrónomos, es necesario compararlas con sus semejantes europeos y norteamericanos. Esto es, con la historia del concepto de herencia en la medicina europea antes de 1900, y con la historia de las aplicaciones de la genética vegetal después de ese año.

De ambas etapas es notorio que el desarrollo de la genética en México se ha dado más por el lado práctico que por el teórico: los médicos se interesan por la herencia porque existen ciertas enfermedades que ellos no son capaces de curar; tales enfer-

57 RHOADES, M.M. (1992), «The early years of maize genetics», FEDOROFF, N. and BOTSTEIN, D. (1992), The Dynamic Genome. Barbara McClintock's ideas in the century of genetics, Cold Spring Harbor Lab. Press. 
medades son recurrentes dentro de una misma familia y algunas incluso se presentan a la misma edad entre los diferentes individuos de generaciones sucesivas; a los agrónomos interesa el mejoramiento de las variedades vegetales de valor económico porque les permitirá obtener mayores rendimientos y los gobiernos mexicanos les apoyan porque están interesados en que los ingresos obtenidos en el ramo agrícola aumenten. Tal vez, este mismo fenómeno se haya dado en otros países de latinoamerica (introducción y desarrollo primario de los aspectos más prácticos de las ciencias) y estaría relacionado directamente, creo, con su situación como países colonizados por largos períodos de tiempo.

Por otra parte, de una manera más puntual, podemos decir de las ideas sobre la herencia en la segunda mitad del siglo pasado (y en general de la medicina mexicana de la época), que sufre en una evolución desde perspectivas más relacionadas con el vitalismo hacia tendencias más reduccionistas, y que este cambio se da de una manera más rápida durante la década de 1870 , en relación con el advenimiento de la política positivista dentro de los altos círculos intelectuales mexicanos. A partir de esos años, existe una promoción muy fuerte de la experimentación para comprobar hipótesis, así como una prefencia por las explicaciones basadas en entidades materiales, aunque una buena dosis de teísmo sigue estando presente.

Aparentemente, la comunidad médica conserva las ideas prevalentes dentro de su tradición en cuanto a la herencia al llegar el siglo XX. Aunque Alfonso L. Herrera incluye ya en 1904 una referencia a la ley de dominancia de Mendel, el contexto en que la incluye es evolutivo, más en el sentido macromutacionista de De Vries, y al parecer, no vislumbra una conexión o implicación directa para la herencia como es considerada por los médicos de su tiempo, y de la cual poseía seguramente un conocimiento bastante cercano (desde que él mismo se desenvuelve y se ha formado dentro de esa misma tradición). Sea porque los médicos no se convencen de la veracidad e implicaciones de la teoría de Mendel, sea porque sus intereses más urgentes se encuentran lejos de los problemas teóricos involucrados en la transmisión de las enfermedades hereditarias, aunado a las constricciones que la propia economía del país durante la revolución aplican a los presupuestos para investigación, el hecho es que los programas sobre investigación genética no son iniciados ni dentro de la medicina, ni dentro de la incipiente biología (a su vez muy influenciada por la tradición médica), al menos no antes que en el campo de la agricultura (1930).

En cuanto a la genética en la agricultura, los programas de investigación son bastante específicos y comprenden principal y casi exclusivamente el mejoramiento de variedades en plantas de interés económico. La comunidad encargada de llevar a cabo dichos programas es la de los ingenieros agrónomos y la gran mayoría de quienes participan en ellos tiene que ser capacitado en Estados Unidos. Esto ocasiona a la larga un gran problema para el desarrollo posterior de la agricultura durante las décadas de 1960 y 1970, pues la orientación capitalista dentro de la cual son entrenados la mayoría de ellos no corresponde a la realidad de la estructura agraria mexicana. 